PROCEEDINGS OF THE

AMERICAN MATHEMATICAL SOCIETY

Volume 125, Number 11, November 1997, Pages 3381-3389

S 0002-9939(97)04220-2

\title{
INCOMPLETENESS OF THE LINEAR SPAN OF THE POSITIVE COMPACT OPERATORS
}

\author{
Z. L. CHEN AND A. W. WICKSTEAD \\ (Communicated by Palle E. T. Jorgensen)
}

\begin{abstract}
We show that even in the case of a Banach lattice $E$ with an order continuous norm (or whose dual has an order continuous norm) the linear span of the positive compact operators on $E$ need not be complete under the regular norm.
\end{abstract}

\section{INTRODUCTION}

We use the standard notation and terminology for Banach spaces and Banach lattices (see for example [4] or [11]). For Banach lattices $E$ and $F$, the symbol $\mathcal{L}(E, F)$ denotes the space of all (linear and continuous) operators from $E$ to $F$ equipped with the standard operator norm: $\|T\|=\sup \{\|T x\|: x \in E,\|x\| \leq 1\}$. Recall that an operator $T \in \mathcal{L}(E, F)$ is regular if $T=T_{1}-T_{2}$ for some positive operators $T_{1}, T_{2}$. As usual, $\mathcal{L}^{r}(E, F)$ denotes the space of all regular operators from $E$ into $F$. With respect to the uniform operator norm the space $\mathcal{L}^{r}$ is not Banach in general, but there exists a natural norm on $\mathcal{L}^{r}$, the regular norm $\|\cdot\|_{r}$, which turns $\mathcal{L}^{r}(E, F)$ into a Banach lattice (see for example [11], Proposition 1.3.6). Namely,

$$
\|T\|_{r}=\inf \left\{\|S\|: S \in \mathcal{L}_{+}(E, F), S \geq \pm T\right\} .
$$

If $F$ is Dedekind complete, then $\mathcal{L}^{r}(E, F)$ is also a Dedekind complete lattice and in this case, $\|T\|_{r}=\||T|\|$.

We will use the notation $\mathcal{K}(E, F)$ to denote the compact linear operators from $E$ into $F$. It is unclear what is the "correct" analogue of $\mathcal{K}(E, F)$ inside $\mathcal{L}^{r}(E, F)$. The only agreement in the literature seems to be that the intersection $\mathcal{K}(E, F) \cap \mathcal{L}^{r}(E, F)$ is not the right object to study. In work on the order essential spectrum, Arendt and Sourour [5], [6] and [7] have made use of the closure in $\mathcal{L}^{r}(E, F)$ of the finite rank operators under the regular norm (see also Raubenheimer's work in [12] and [13]). In studying the order structure of spaces of compact operators [1], [2] and [3], Abramovich and Wickstead proposed studying the linear span of the positive compact operators, which we denote by $\mathcal{K}^{r}(E, F)$. Whilst this space has the merit of being spanned by its positive cone (which is not the case for $\mathcal{K}(E, F) \cap \mathcal{L}^{r}(E, F)$, see [1]) it still need not be lattice ordered [3], which is what results of Krengel [9] and [10] might have led one to conjecture. In this note we show that $\mathcal{K}^{r}(E, F)$ also fails, in general, to be closed in $\mathcal{L}^{r}(E, F)$ for the regular norm. Apart from other

Received by the editors June 26, 1996.

1991 Mathematics Subject Classification. Primary 47B65; Secondary 47 B07.

Key words and phrases. Compact operators, regular operators.

(C)1997 American Mathematical Society 
considerations, this makes it unlikely that it can be used to develop an alternative essential order spectral theory. In a concluding section, we show that $\mathcal{K}^{r}(E, F)$ need not have the Riesz Separation Property, answering a question posed in [2].

Let us point out now that if the pair $(E, F)$ has the compact domination property (i.e. $0 \leq S \leq T \in \mathcal{K}(E, F) \Rightarrow S \in \mathcal{K}(E, F)$, see [8] and [15]) and $\mathcal{L}^{r}(E, F)$ is a lattice (or even if it has the Riesz separation property, see [14]) then $\mathcal{K}^{r}(E, F)$ is closed in $\mathcal{L}^{r}(E, F)$ under the regular norm. In particular our counterexample cannot satisfy the Dodds-Fremlin conditions that both $E^{*}$ and $F$ have order continuous norms. However we will be able to give counterexamples in which either $E^{*}$ or $F$ has an order continuous norm. Let us note in passing that Example 3.7 in [5] shows that even when the Dodds-Fremlin conditions (and the approximation property) hold then $\mathcal{K}^{r}(E, F)$ is not equal to the regular-norm closure of the finite rank operators.

\section{NORM INCOMPLETENESS}

The construction of our examples will be by means of a new norm that can be defined on $\mathcal{K}^{r}(E, F)$. Under this norm $\mathcal{K}^{r}(E, F)$ is norm complete. This norm dominates the regular norm and if $\mathcal{K}^{r}(E, F)$ were complete under the regular norm then the two norms would be equivalent. We complete the argument by showing that they need not be.

Definition 2.1. If $E$ and $F$ are Banach lattices then the $k$-norm on $\mathcal{K}^{r}(E, F)$ is defined by

$$
\|T\|_{k}=\inf \left\{\|S\|: S \in \mathcal{K}_{+}(E, F), S \geq \pm T\right\} .
$$

It is clear that this does define a norm on $\mathcal{K}^{r}(E, F)$ and that $\|T\|_{k} \geq\|T\|_{r}$ for each $T \in \mathcal{K}^{r}(E, F)$.

Proposition 2.2. For any Banach lattices $E$ and $F, \mathcal{K}^{r}(E, F)$ is complete under the k-norm.

Proof. Let $\left(T_{n}\right)$ be a $\|\cdot\|_{k^{-}}$Cauchy sequence in $\mathcal{K}^{r}(E, F)$. By passing to a subsequence we may suppose that for each $n \in \mathbb{N}$ we have $\left\|T_{n}-T_{n+1}\right\|_{k}<2^{-n}$. Standard arguments show that it suffices to prove that this subsequence converges. As $\|U\| \leq\|U\|_{k}$ for all $U \in \mathcal{K}^{r}(E, F)$ the sequence $\left(T_{n}\right)$ is certainly Cauchy in the operator norm so that $T_{n} \rightarrow T \in \mathcal{K}(E, F)$. By definition of the $k$-norm, there are $U_{n} \in \mathcal{K}_{+}(E, F)$ with $\pm\left(T_{n}-T_{n+1}\right) \leq U_{n}$ and $\left\|U_{n}\right\|<2^{-n}$ for all $n \in \mathbb{N}$. Let $Q_{n}=\sum_{k=n}^{\infty} U_{k}$ so that $Q_{n}$ is compact and positive and $\left\|Q_{n}\right\| \leq 2^{-n+1}$. Thus for each $x \in E_{+}$we have

$$
\begin{aligned}
\left|\left(T_{n}-T\right) x\right| & =\lim _{m \rightarrow \infty}\left|\left(T_{n}-T_{m}\right) x\right| \\
& \leq \lim _{m \rightarrow \infty} \sum_{k=n}^{m-1}\left|\left(T_{k}-T_{k+1}\right) x\right| \\
& \leq \lim _{m \rightarrow \infty} \sum_{k=n}^{m-1} U_{k} x \\
& =Q_{n} x
\end{aligned}
$$

I.e. $\pm\left(T_{n}-T\right) \leq Q_{n}$. One immediate consequence of this inequality is that $T_{n}-T \in$ $\mathcal{K}^{r}(E, F)$ and hence $T \in \mathcal{K}^{r}(E, F)$. Another is that $\left\|T_{n}-T\right\|_{k} \leq\left\|Q_{n}\right\|<2^{-n+1}$ 
so that $T$ is the required limit of the sequence $\left(T_{n}\right)$ and hence $\mathcal{K}^{r}(E, F)$ is indeed complete under the $k$-norm.

Routine arguments will show that if $E=F$ then $\mathcal{K}^{r}(E)$ is a Banach algebra under the $k$-norm and that if $K^{r}(E, F)$ is a lattice then it is a Banach lattice (even if it is not, as still seems possibly may happen, a sublattice of $\mathcal{L}^{r}(E, F)$ ).

The construction of our example will make use of ideas from [3]. We will also make use of the Rademacher functions $r_{n}$ which may be defined on $[0,1]$ by defining $r_{n}(t)$ to be the sign of $\sin \left(2^{n} \pi t\right)$. Before giving details of our construction, let us establish some properties of the Rademacher functions that do not seem readily accessible in the literature in quite the form that we want.

Lemma 2.3. Let $0 \leq p_{1}<p_{2}<\cdots<p_{m}$ be positive integers and let $k \geq 0$ be an integer. If $n$ and $j$ are integers with $n \geq \max \{k, 1\}$ and $0 \leq j \leq 2^{k}-1$ then

$$
\int_{\left[j / 2^{k},(j+1) / 2^{k}\right]} r_{n+p_{1}}(t) r_{n+p_{2}}(t) \cdots r_{n+p_{m}}(t) d t=0 .
$$

Proof. The proof will be by induction on $m$. In the case $m=1$ we need only note that

$$
\int_{\left[j / 2^{k},(j+1) / 2^{k}\right]} r_{n+p_{1}}(t) d t=\frac{1}{2^{k}} \int_{[0,1]} r_{n+p_{1}-k}(t) d t=0 .
$$

Suppose the result is known for $m=m_{0}$; then we will establish it for $m=m_{0}+$ 1. The interval $I=\left[j / 2^{k},(j+1) / 2^{k}\right]$ may be divided into $2^{n+p_{1}-k}$ intervals $I_{p}$ (say) where each $I_{p}$ is of the form $\left[\ell / 2^{n+p_{1}},(\ell+1) / 2^{n+p_{1}}\right]$ for $j 2^{n+p_{1}-k} \leq \ell<$ $(j+1) 2^{n+p_{1}-k}$. Note that $r_{n+p_{1}}$ is identically either 1 or -1 on each $I_{p}$ (apart from at endpoints), so that

$$
\begin{aligned}
\int_{I} r_{n+p_{1}}(t) r_{n+p_{2}}(t) & \cdots r_{n+p_{m_{0}+1}}(t) d t \\
& =\sum_{p=1}^{2^{n+p_{1}-k}-1} \int_{I_{p}} r_{n+p_{1}}(t) r_{n+p_{2}}(t) \cdots r_{n+p_{m_{0}+1}}(t) d t \\
& =\sum_{p=1}^{2^{n+p_{1}-k}-1}( \pm 1) \int_{I_{p}} r_{n+p_{2}}(t) \cdots r_{n+p_{m_{0}+1}}(t) d t
\end{aligned}
$$

Applying the inductive hypothesis to the sequence $\left(p_{2}-p_{1}, p_{3}-p_{1}, \ldots, p_{m_{0}+1}-p_{1}\right)$ and with $n$ replaced by $n+p_{1}$ we see that each

$$
\sum_{p=1}^{2^{n+p_{1}-k}-1}( \pm 1) \int_{I_{p}} r_{n+p_{2}}(t) \cdots r_{n+p_{m_{0}+1}}(t) d t=0
$$

and therefore

$$
\int_{I} r_{n+p_{1}}(t) r_{n+p_{2}}(t) \cdots r_{n+p_{m_{0}+1}}(t) d t=0
$$

completing the inductive proof.

Lemma 2.4. Let $0 \leq p_{1}<p_{2}<\cdots<p_{m}$ be positive integers. Then

$$
r_{n+p_{1}} r_{n+p_{2}} \cdots r_{n+p_{m}} \rightarrow 0 \text { weakly in } L_{2}[0,1] .
$$


Proof. Let $h_{n}=r_{n+p_{1}} r_{n+p_{2}} \cdots r_{n+p_{m}}$, so that it suffices to show that $\left\langle h_{n} \mid \chi_{(a, b)}\right\rangle \rightarrow$ 0 as $n \rightarrow \infty$ whenever $(a, b) \subset[0,1]$, where $\langle\cdot \mid \cdot\rangle$ denotes the usual inner product on $L_{2}[0,1]$. If $\epsilon>0$, choose positive integers $i, j, k$ such that $a \leq i / 2^{k}<a+\epsilon$ and $b-\epsilon<j / 2^{k} \leq b$. We then have

$$
\begin{aligned}
\left|\left\langle h_{n} \mid \chi_{(a, b)}\right\rangle\right| & =\left|\left\langle h_{n} \mid \chi_{\left(a, i / 2^{k}\right)}\right\rangle+\sum_{p=i}^{j-1}\left\langle h_{n} \mid \chi_{\left(p / 2^{k},(p+1) / 2^{k}\right)}\right\rangle+\left\langle h_{n} \mid \chi_{\left(j / 2^{k}, b\right)}\right\rangle\right| \\
& =\left|\left\langle h_{n} \mid \chi_{\left(a, i / 2^{k}\right)}\right\rangle+\left\langle h_{n} \mid \chi_{\left(j / 2^{k}, b\right)}\right\rangle\right| \\
& \leq 2 \epsilon
\end{aligned}
$$

provided $n \geq k$. We thus certainly have $\left\langle h_{n} \mid \chi_{(a, b)}\right\rangle \rightarrow 0$ and therefore $h_{n} \rightarrow 0$ weakly.

Proposition 2.5. Fix a positive integer $m$ and let $g_{n}=r_{n}^{+} r_{n+1}^{+} \cdots r_{n+m-1}^{+}$. Then $g_{n} \rightarrow 2^{-m}$ weakly and $\left\|g_{n}\right\|_{2}=2^{-m / 2}$ for all $n \in \mathbb{N}$.

Proof. Note that for each $p \in \mathbb{N}, r_{p}^{+}=\left(1+r_{p}\right) / 2$. Thus we may write

$$
g_{n}=2^{-m}\left(1+r_{n}\right)\left(1+r_{n+1}\right)\left(1+r_{n+m-1}\right)=2^{-m}\left(1+H_{n}\right)
$$

where $H_{n}$ is a sum of terms of the form $r_{n+p_{1}} \cdots r_{n+p_{j}}$ each of which converges weakly to 0 by Lemma 2.4. Thus $H_{n} \rightarrow 0$ and hence $g_{n} \rightarrow 2^{-m}$ as claimed. It follows from Lemma 2.3 that $\int_{0}^{1} H_{n}(t) d t=0$ so, noting that $g_{n}=g_{n}^{2}$, we have

$$
\begin{aligned}
\left\|g_{n}\right\|_{2} & =\left(\int_{0}^{1} g_{n}(t)^{2} d t\right)^{1 / 2} \\
& =\left(\int_{0}^{1} g_{n}(t) d t\right)^{1 / 2} \\
& =\left(\int_{0}^{1} 2^{-m}\left(1+H_{n}(t)\right) d t\right)^{1 / 2} \\
& =\left[2^{-m}\left(1+\int_{0}^{1} H_{n}(t) d t\right)\right]^{1 / 2} \\
& =2^{-m / 2} .
\end{aligned}
$$

We need now to introduce a notion of approximate inequality that seems to make the presentation of our proofs much easier.

Lemma 2.6. If $E$ is a Banach lattice and $v, f \in E$ then the following are equivalent:

(i) $\exists w \in E$ with $\|f-w\|<\epsilon$ and $w \geq v$,

(ii) $\left\|(f-v)^{-}\right\|<\epsilon$.

Proof. If (i) holds then $w \geq v \Rightarrow-v \geq-w \Rightarrow f-v \geq f-w \Rightarrow(f-v)^{-} \leq$ $(f-w)^{-} \Rightarrow\left\|(f-v)^{-}\right\| \leq\left\|(f-w)^{-}\right\| \leq\|f-w\|<\epsilon$. If, on the other hand, (ii) holds, simply taking $w=(f \vee v)$ we clearly have $w \geq v$ and $w-f=(f-v)^{-}$so that the norm condition is clear. 
We will denote the above relationship between $v$ and $f$ by $f \geq_{\llbracket \epsilon \rrbracket} v$. Note that if $f \geq_{\llbracket \epsilon_{1} \rrbracket} a$ and $f \geq_{\llbracket \epsilon_{2} \rrbracket} b$ then we certainly have $f \geq_{\llbracket \epsilon_{1}+\epsilon_{2} \rrbracket} a \vee b$ and that if $\lambda>0$ then $\lambda f \geq_{\llbracket \lambda \epsilon_{1} \rrbracket} \lambda a$. It is also easy to see that if $f \geq_{\llbracket \epsilon \rrbracket} v$ and $v \geq 0$ then $\|f\| \geq\|v\|-\epsilon$.

The following proposition brings together the two essential elements of our main proof, namely sequences of positive parts of Rademacher functions and the relationship $\geq_{\llbracket \epsilon \rrbracket}$.

Proposition 2.7. Let $r_{n}$ denote the $n$ 'th Rademacher function and fix $m \in \mathbb{N}$. Let $g_{n}=r_{n}^{+} r_{n+1}^{+} \cdots r_{n+m-1}^{+}, f \in L_{2}[0,1], \epsilon>0$ and $p \in \mathbb{N}$. If there is a subsequence $\left(g_{n_{k}}\right)$ such that $f \geq \llbracket \epsilon \rrbracket g_{n_{k}}$ for all $k \in \mathbb{N}$ then $f \geq \llbracket p \in \rrbracket\left[1-\left(1-2^{-m}\right)^{p}\right]$.

Proof. The proof will be by induction on $p$. We first prove it for the case $p=1$. Let $w_{k}$ denote an element of $L_{2}[0,1]$ with $w_{k} \geq g_{n_{k}}$ and $\left\|f-w_{k}\right\|_{2}<\epsilon$. We denote the usual inner product on $L_{2}[0,1]$ by $\langle\cdot \mid \cdot\rangle$. For each $k \in \mathbb{N}$ and $0 \leq u \in L_{2}[0,1]$ with $\|u\|_{2} \leq 1$ we have

$$
\begin{aligned}
\langle f \mid u\rangle & =\left\langle f-w_{k} \mid u\right\rangle+\left\langle w_{k} \mid u\right\rangle \\
& \geq-\epsilon+\left\langle g_{n_{k}} \mid u\right\rangle \\
& \rightarrow-\epsilon+\left\langle 2^{-m}, u\right\rangle
\end{aligned}
$$

by Lemma 2.5. I.e. $\left\langle f-2^{-m}, u\right\rangle \geq-\epsilon$ whenever $u \in L_{2}[0,1]_{+}$and $\|u\|_{2} \leq 1$. It follows that $\left\|\left(f-2^{-m}\right)^{-}\right\|_{2}<\epsilon$; by taking $u=\frac{\left(f-2^{-m}\right)^{-}}{\left\|\left(f-2^{-m}\right)^{-}\right\|_{2}}$ we see that

$$
\left\langle f-2^{-m} \mid u\right\rangle=\left\langle\left(f-2^{-m}\right)^{+} \mid u\right\rangle-\left\langle\left(f-2^{-m}\right)^{-} \mid u\right\rangle=-\left\|(f-u)^{-}\right\|_{2}
$$

(except when $\left(f-2^{-m}\right)^{-}=0$, when there is nothing to prove). I.e. $f \geq \llbracket € \rrbracket$ $\left[1-\left(1-2^{-m}\right)^{1}\right]$ as claimed.

Now suppose that for some $q \in \mathbb{N}$ we have $f \geq_{\llbracket q \epsilon \rrbracket}\left[1-\left(1-2^{-m}\right)^{q}\right]$ as well as $f \geq \llbracket \epsilon \rrbracket g_{n_{k}}$ for some infinite subsequence $\left(g_{n_{k}}\right)$. It follows, as noted after Lemma 2.6 , that $f \geq \llbracket(q+1) \epsilon \rrbracket\left(\left[1-\left(1-2^{-m}\right)^{q}\right] \vee g_{n_{k}}\right.$ for each $k \in \mathbb{N}$. Note that since each $g_{n_{k}}$ takes only the values 0 and $1,\left[1-\left(1-2^{-m}\right)^{q}\right] \vee g_{n_{k}}=\left(1-2^{-m}\right)^{q} g_{n_{k}}+\left[1-\left(1-2^{-m}\right)^{q}\right]$. It follows that $\left.\left(1-2^{-m}\right)^{-q}\left(f-\left[1-\left(1-2^{-m}\right)^{q}\right]\right)\right) \geq \llbracket\left(1-2^{-m}\right)^{-q}(q+1) \epsilon \rrbracket g_{n_{k}}$ for each $k \in \mathbb{N}$. Applying the case $p=1$, which we have already proved, to the function $\left(1-2^{-m}\right)^{-q}\left(f-\left[1-\left(1-2^{-m}\right)^{q}\right]\right)$ we see that

$$
\left(1-2^{-m}\right)^{-q}\left(f-\left[1-\left(1-2^{-m}\right)^{q}\right]\right) \geq \llbracket\left(1-2^{-m}\right)^{-q}(q+1) \epsilon \rrbracket 2^{-m} .
$$

Using the properties of $\geq_{\llbracket \epsilon \rrbracket}$ noted after Lemma 6 we thus have

$$
f \geq \llbracket(q+1) \epsilon \rrbracket\left[1-\left(1-2^{-m}\right)^{q}+2^{-m}\left(1-2^{-m}\right)^{q}\right]=\left[1-\left(1-2^{-m}\right)^{q+1}\right],
$$

completing the proof.

Theorem 2.8. Let $E=\ell_{1}\left(\ell_{2}^{2^{n}}\right)$ and $F=L_{2}[0,1]$. For each $m \in \mathbb{N}$ there is an operator $T_{m} \in \mathcal{K}^{r}(E, F)$ with $\left\|T_{m}\right\|_{r}=2^{-m / 2}$ and $\left\|T_{m}\right\|_{k}=1$.

Proof. Let $S_{n}$ denote an operator on $\ell_{2}^{2^{n}}$ with $\left\|S_{n}\right\|=2^{n / 2}$ and with all entries in the matrix representing $\left|S_{n}\right|$ (for the standard basis) equal to 1 and therefore $\left\|\left|S_{n}\right|\right\|=2^{n}$. These matrices were first introduced into the subject by Krengel and an account of them may be found in [4] on page 270. $J_{n}: \ell_{2}^{2^{n}} \rightarrow L_{2}[0,1]$ will be defined by $J_{n}\left(x_{1}, x_{2}, \ldots, x_{2^{n}}\right)=\sum_{k=1}^{2^{n}} x_{k} \chi_{\left[(k-1) 2^{-n}, k 2^{-n}\right]}$. As was pointed out in [3], $\left\|J_{n} \circ S_{n}\right\|=1$ whilst $\left\|\left|J_{n} \circ S_{n}\right|\right\|=\left\|J_{n} \circ\left|S_{n}\right|\right\|=2^{n / 2}$. We also noted that $J_{n} \circ\left|S_{n}\right|\left(x_{1}, x_{2}, \ldots, x_{2^{n}}\right)=\left(\sum_{k=1}^{2^{n}} x_{k}\right) \chi_{[0,1]}$. 
Let $g_{n}=r_{n}^{+} r_{n+1}^{+} \cdots r_{n+m-1}^{+}$and define $T_{m}: E \rightarrow F$ by

$$
T_{m}\left(\mathbf{x}_{1}, \mathbf{x}_{2}, \ldots\right)=\sum_{n=1}^{\infty} 2^{-n / 2} g_{n} J_{n} S_{n} \mathbf{x}_{n}
$$

where we use $\mathbf{x}_{n}$ to denote an element of $\ell_{2}^{2^{n}}$. As was pointed out in [2], $T_{m}$ is a compact operator which has a modulus (in $\mathcal{L}^{r}(E, F)$ ) and

$$
\left|T_{m}\right|\left(\mathbf{x}_{1}, \mathbf{x}_{2}, \ldots\right)=\sum_{n=1}^{\infty} 2^{-n / 2} g_{n} J_{n}\left|S_{n}\right| \mathbf{x}_{n} .
$$

Define also $U: E \rightarrow F$ by

$$
U\left(\mathbf{x}_{1}, \mathbf{x}_{2}, \ldots\right)=\sum_{n=1}^{\infty} 2^{-n / 2} J_{n} S_{n} \mathbf{x}_{n}
$$

which is compact and with $\left|T_{m}\right| \leq U$ for each $m \in \mathbb{N}$. We thus certainly have $T_{m} \in \mathcal{K}^{r}(E, F)$.

Note that $\left\|g_{n}\right\|_{2}=2^{-m / 2}$, so that

$$
\begin{aligned}
\left\|\left|T_{m}\right|\left(\mathbf{x}_{n}\right)\right\|_{2} & =\left\|\sum_{n=1}^{\infty} 2^{-n / 2} g_{n} J_{n}\left|S_{n}\right| \mathbf{x}_{n}\right\|_{2} \\
& \leq \sum_{n=1}^{\infty} 2^{-n / 2}\left\|g_{n}\right\|_{2}\left\|J_{n} \circ\left|S_{n}\right|\right\|\left\|\mathbf{x}_{n}\right\| \\
& =2^{-m / 2} \sum_{n=1}^{\infty}\left\|\mathbf{x}_{n}\right\| \\
& =2^{-m / 2}\left\|\left(\mathbf{x}_{n}\right)\right\|
\end{aligned}
$$

for all $\left(\mathbf{x}_{n}\right) \in E$, so that $\left\|\left|T_{m}\right|\right\| \leq 2^{-m / 2}$. The converse inequality is readily established, so that $\left\|\left|T_{m}\right|\right\|=2^{-m / 2}$.

The operator $U$ defined above shows that $\left\|T_{m}\right\|_{k} \leq 1$. We show next that any compact operator $V \geq \pm S$ (and hence with $V \geq|S|$ ) must also have operator norm of at least 1 and hence $\left\|T_{m}\right\|_{k}=1$.

For each $n \in \mathbb{N}$ let $\overline{\mathbf{x}}^{n}$ denote that element of $\ell_{1}\left(\ell_{2}^{2^{n}}\right)$ with all entries zero apart from the $n$ 'th which is $x_{n}=\left(2^{-n / 2}\right)_{1}^{2^{n}}$. Each $\overline{\mathbf{x}}^{n}$ has norm 1 and its image under $\left|T_{m}\right|$ is $g_{n}$. It follows that $V\left(\overline{\mathbf{x}}^{n}\right) \geq g_{n}$ for each $n \in \mathbb{N}$. As $V$ is assumed to be compact, the set $A=\left\{V\left(\overline{\mathbf{x}}^{n}\right): n \in \mathbb{N}\right\}$ is a totally bounded subset of $L_{2}[0,1]$. If $\eta>0$ choose $p \in \mathbb{N}$ such that $\left(1-2^{-m}\right)^{p}<\eta / 2$ and then choose $\epsilon>0$ such that $\epsilon<\eta /(2 p)$. Now let $\left\{v_{1}, v_{2}, \ldots, v_{m}\right\}$ be a subset of $A$ which is an $\epsilon$-net for $A$. For each $n \in \mathbb{N}$ there is $k_{n} \in\{1,2, \ldots, m\}$ such that $\left\|v_{k_{n}}-V\left(\overline{\mathbf{x}}^{n}\right)\right\|_{2}<\epsilon$ so that $v_{k_{n}} \geq \llbracket \epsilon \rrbracket g_{n}$. As there are finitely many choices of $k_{n}$ and infinitely many $n$, there is a choice of $k_{0} \in\{1,2, \ldots, m\}$ with $v_{k_{0}} \geq \llbracket \epsilon \rrbracket g_{n_{k}}$ for infinitely many $k \in \mathbb{N}$. It follows from Proposition 2.7 that $v_{k_{0}} \geq_{\llbracket p \epsilon \rrbracket}\left[1-\left(1-2^{-m}\right)^{p}\right]$. In particular it follows that

$$
\begin{aligned}
\left\|v_{k_{0}}\right\|_{2} & \geq\left\|1-\left(1-2^{-m}\right)^{p}\right\|_{2}-p \epsilon \\
& =1-\left(1-2^{-m}\right)^{p}-p \epsilon \\
& >1-\eta / 2-\eta / 2 \\
& =1-\eta .
\end{aligned}
$$


Since $v_{k_{0}} \in A$ and is therefore the image under $V$ of a member of the unit ball of $\ell_{1}\left(\ell_{2}^{2^{n}}\right)$, the norm of $V$ is at least $1-\eta$. This argument applies for all $\eta>0$ so that $\|V\| \geq 1$ and hence $\|S\|_{k} \geq 1$ as claimed.

Corollary 2.9. $\mathcal{K}^{r}\left(\ell_{1}\left(\ell_{2}^{2^{n}}\right), L_{2}[0,1]\right)$ is not complete under the regular norm.

Proof. If it were, then Banach's isomorphism theorem would tell us that the regular norm is equivalent to the $k$-norm, which would contradict Theorem 2.8.

In this example, the range space has an order continuous norm. By taking adjoints we obtain an example where the domain has a dual with order continuous norm. The proof is virtually the same as that of Theorem 2.8 and Corollary 2.9, using Theorem 5.11 of [4] to see that with these spaces we always have $|T|^{*}=\left|T^{*}\right|$ for any regular operator $T$. We omit the details.

Corollary 2.10. $\mathcal{K}^{r}\left(L_{2}[0,1], \ell_{\infty}\left(\ell_{2}^{2^{n}}\right)\right)$ is not complete under the regular norm.

To conclude this section, let us pose the problem of whether or not it is possible for $\mathcal{K}^{r}(E, F)$ to be complete under the regular norm but with the regular norm not equal to the $k$-norm. I.e. can the two norms be equivalent without being equal?

\section{Failure of the Riesz Separation Property}

Recall that an ordered space has the Riesz Separation Property (we abbreviate this to RSP in future) if whenever $x_{1}, x_{2} \leq z_{1}, z_{2}$ there is $y$ with $x_{1}, x_{2} \leq y \leq z_{1}, z_{2}$. Lattices clearly have the RSP (take $y=x_{1} \vee x_{2}$, for example) but there are nonlattices with the RSP. In [14], one of us showed that it is possible for the space of all regular operators between two Banach lattices to have the Riesz Separation Property (RSP) without being a lattice and also that such a space of operators need not have the RSP. In [2], the question was posed of whether or not the same possibilities could occur for $\mathcal{K}^{r}(E, F)$. The example in [14] cannot easily be modified to provide the desired compactness, but we can now show that the construction used in [3] may be modified to give an example of a space of compact operators without the RSP. This example also answers the corresponding question for weakly compact operators and for operators with the Dunford-Pettis property. The question of whether or not the linear span of the positive compact operators can have the RSP without being a lattice remains open.

As well as the operators $J_{n}: \ell_{2}^{2^{n}} \rightarrow L_{2}[0,1]$ and $S_{n}: \ell_{2}^{2^{n}} \rightarrow \ell_{2}^{2^{n}}$ introduced in the proof of Theorem 2.8, we will make use of the operator $Q_{n}: L_{2}[0,1] \rightarrow H_{n}=$ $J_{n}\left(\ell_{2}^{2^{n}}\right)$ which is defined by

$$
Q_{n}: f \mapsto 2^{n} \sum_{k=1}^{2^{n}}\left(\int_{\frac{k-1}{2^{n}}}^{\frac{k}{2^{n}}} f d \mu\right) \chi_{\left[\frac{k-1}{2^{n}}, \frac{k}{2^{n}}\right]},
$$

where $\mu$ denotes Lebesgue measure on $[0,1]$. This is a contractive projection. It was established in [3] that $\left\|J_{n} \circ S_{n} \circ J_{n}^{-1} \circ Q_{n}\right\|=2^{n / 2}$ whilst $\left\|\mid J_{n} \circ S_{n} \circ J_{n}^{-1} \circ Q_{n}\right\|=$ $\left\|J_{n} \circ\left|S_{n}\right| \circ J_{n}^{-1} \circ Q_{n}\right\|=2^{n}$. Note also that $2^{-n} J_{n} \circ\left|S_{n}\right| \circ J_{n}^{-1} \circ Q_{n} f=\left(\int_{0}^{1} d \mu\right) \chi_{[0,1]}$ for all $f \in L_{2}[0,1]$.

Our example uses the spaces $E=L_{2}[0,1]$ and $F=c\left(L_{2}[0,1]\right)$, the Banach lattice of all convergent sequences $\left(f_{1}, f_{2}, \ldots\right)$ of functions from $L_{2}$ normed by

$$
\left\|\left(f_{1}, f_{2}, \ldots\right)\right\|_{c\left(L_{2}[0,1]\right)}=\sup _{n \in \mathbb{N}}\left\|f_{n}\right\|_{L_{2}}
$$


Let $\left(\alpha_{n}\right)$ and $\left(\beta_{n}\right)$ be any sequences of reals with $\left|\alpha_{2 n}\right|=\left|\beta_{2 n-1}\right|=1$ and $\alpha_{2 n-1}=\beta_{2 n}=0$ for all $n \in \mathbb{N}$. Define operators $T, U, V: E \rightarrow F$ by

$$
\begin{aligned}
T f & =\left(\alpha_{n} 2^{-n} J_{n} S_{n} J_{n}^{-1} Q_{n} f\right)_{n=1}^{\infty}, \\
U f & =\left(\beta_{n} 2^{-n} J_{n} S_{n} J_{n}^{-1} Q_{n} f\right)_{n=1}^{\infty}, \\
V f & =\left(2^{-n} J_{n}\left|S_{n}\right| J_{n}^{-1} Q_{n} f\right)_{n=1}^{\infty}=\left(\int_{0}^{1} f d \mu\right)\left(\chi_{[0,1]}, \chi_{[0,1]}, \ldots\right) .
\end{aligned}
$$

It is clear that all three operators do take values in $F$, the last taking values that are constant sequences and the first two having values that converge to zero in norm. Other properties of these three operators are contained in the following result.

Proposition 3.1. With the notation above, we have

(i) $T, U$ and $V$ are compact operators.

(ii) $\pm T, \pm U \leq V$ so that $T, U, V \in \mathcal{K}^{r}(E, F)$.

(iii) $-T, T \leq V-U, V+U$.

(iv) If $W \in L(E, F)$ with $W \geq \pm T$ then

$$
(W f)_{2 n} \geq 2^{-2 n} J_{2 n}\left|S_{2 n}\right| J_{2 n}^{-1} Q_{2 n} f=\left(\int_{0}^{1} f d \mu\right) \chi_{[0,1]}
$$

for all $f \in E_{+}$.

(v) If $W \in L(E, F)$ with $W \geq \pm U$ then

$$
(W f)_{2 n-1} \geq 2^{-(2 n-1)} J_{2 n-1}\left|S_{2 n-1}\right| J_{2 n-1}^{-1} Q_{2 n-1} f=\left(\int_{0}^{1} f d \mu\right) \chi_{[0,1]}
$$

for all $f \in E_{+}$.

Proof. Parts (i), (ii) and (iii) are immediate from their definitions. We prove (iv), the proof of $(\mathrm{v})$ being almost identical.

If $W \geq \pm T$ and $f \in E_{+}$, let $|g| \leq f$. Then we see that

$$
\begin{gathered}
(W \pm T) g^{+}=W g^{+} \pm T g^{+} \geq 0, \\
(W \pm T) g^{-}=W g^{-} \pm T g^{-} \geq 0
\end{gathered}
$$

so that $W|g| \geq \pm T g$. It follows that $W|g| \geq|T g|$ so that

$$
(W f)_{2 n} \geq(W|g|)_{2 n} \geq|T g|_{2 n}=\left|2^{-2 n} J_{2 n} S_{2 n} J_{2 n}^{-1} Q_{2 n} g\right| \text {. }
$$

Taking the supremum over all such $g$, we see that

$$
\begin{aligned}
(W f)_{2 n} & \geq\left|2^{-2 n} J_{2 n} S_{2 n} J_{2 n}^{-1} Q_{2 n}\right|(f) \\
& =2^{-2 n} J_{2 n}\left|S_{2 n}\right| J_{2 n}^{-1} Q_{2 n}(f) \\
& =\left(\int_{0}^{1} f d \mu\right) \chi_{[0,1]}
\end{aligned}
$$

which establishes (iv).

Theorem 3.2. The space $\mathcal{K}^{r}\left(L_{2}[0,1], c\left(L_{2}[0,1]\right)\right)$ does not have the Riesz Separation Property.

Proof. With the notation in use above, we show that there is no linear operator $W$ with $-T, T \leq W \leq V-U, V+U$. If there were then part (iv) of Proposition 3.1 establishes that $\left(W \chi_{[0,1]}\right)_{2 n} \geq \chi_{[0,1]}$ for all $n \in \mathbb{N}$, whilst part (v) shows that $\left(V \chi_{[0,1]}-W \chi_{[0,1]}\right)_{2 n-1} \geq \chi_{[0,1]}$. We know that $\left(V \chi_{[0,1]}\right)_{2 n-1}=\chi_{[0,1]}$, so that 
$\left(W \chi_{[0,1]}\right)_{2 n-1} \leq 0$. If the sequence $W \chi_{[0,1]}$ converges to a limit $\ell \in L_{2}[0,1]$ then consideration of the subsequences of even and odd terms shows that $0 \geq \ell \geq \chi_{[0,1]}$ which is impossible. Thus no such operator $W$ can exist and hence the space does not have the RSP.

Note that the last proof did not assume any property of $W$ beyond linearity and also that the compact operators $T, U$ and $V$ are certainly weakly compact and have the Dunford-Pettis property, so this example also shows shows that the linear span of either the positive weakly compact operators or of the positive Dunford-Pettis operators need not have the RSP.

\section{REFERENCES}

[1] Y. A. Abramovich and A. W. Wickstead, A compact regular operator without modulus, Proc. Amer. Math. Soc. 116 (1992), 721-726. MR 93a:47038

[2] Y.A. Abramovich and A.W. Wickstead, Recent results on the order structure of compact operators, Irish Math. Soc. Bulletin 32 (1994), 32-45. MR 95h:47050

[3] Y. A. Abramovich and A. W. Wickstead, Solutions of several problems in the theory of compact positive operators, Proc. Amer. Math. Soc. 123 (1995), 3021-3026. MR 95m:47059

[4] C. D. Aliprantis and O. Burkinshaw, Positive Operators, Academic Press, New York \& London, 1985. MR 87h:47086

[5] W. Arendt, On the o-spectrum of regular operators and the spectrum of measures, Math.Z. 178 (1981), 271-287. MR 83h:47027

[6] W. Arendt and A.R. Sourour, Ideals of regular operators on $\ell^{2}$, Proc. Amer. Math. Soc. 88 (1983), 93-96. MR 84c:47048

[7] W. Arendt and A.R. Sourour, Perturbation of regular operators and the order essential spectrum, Indag. Math. 48 (1986), 109-122. MR 87j:47052

[8] P.G. Dodds and D.H. Fremlin, Compact operators in Banach lattices, Israel J. Maths. 34 (1979), 287-320. MR 81g:47037

[9] U. Krengel, Über den Absolutbetrag stetiger linearer Operatoren und seine Anwendung auf ergodische Zerlegungen, Math. Scand. 13 (1963), 151-187. MR 31:310

[10] U. Krengel, Remark on the modulus of compact operators, Bull. Amer. Math. Soc. 72 (1966), 132-133. MR 32:8162

[11] P. Meyer-Nieberg, Banach Lattices, Springer-Verlag, Berlin Heidelberg New York, 1991. MR 93f: 46025

[12] H. Raubenheimer, The o-spectrum of r-asymptotically quasi-finite rank operators, Quaest. Math. 7 (1984), 299-303. MR 86c:47050

[13] H. Raubenheimer, R-asymptotically quasi-finite rank operators and the spectrum of measures,, Quaest. Math. 10 (1986), 97-111. MR 87m:47090

[14] A.W. Wickstead, Spaces of operators with the Riesz separation property, Indag. Math. N.S. 6 (1995), 235-245. MR 96g:47032

[15] A.W. Wickstead, Converses for the Dodds-Fremlin and Kalton-Saab theorems, Math. Proc. Camb. Phil. Soc. 120 (1996), 175-179. MR 96m:47067

Department of Pure Mathematics, The Queen's University of Belfast, Belfast Bt7 1NN, NORTHERN IRELAND

E-mail address: A.Wickstead@qub.ac.uk 